BNL-96363-2011-IR

\title{
Initial Configuration of Acceleration for the IDS-NF Neutrino Factory
}

\author{
J. Scott Berg
}

September 2011

Physics Department

\section{Brookhaven National Laboratory}

\section{U.S. Department of Energy Office of Science}

Notice: This manuscript has been authored by employees of Brookhaven Science Associates, LLC under Contract No. DE-AC02-98CH10886 with the U.S. Department of Energy. The publisher by accepting the manuscript for publication acknowledges that the United States Government retains a non-exclusive, paid-up, irrevocable, world-wide license to publish or reproduce the published form of this manuscript, or allow others to do so, for United States Government purposes. 


\section{DISCLAIMER}

This report was prepared as an account of work sponsored by an agency of the United States Government. Neither the United States Government nor any agency thereof, nor any of their employees, nor any of their contractors, subcontractors, or their employees, makes any warranty, express or implied, or assumes any legal liability or responsibility for the accuracy, completeness, or any third party's use or the results of such use of any information, apparatus, product, or process disclosed, or represents that its use would not infringe privately owned rights. Reference herein to any specific commercial product, process, or service by trade name, trademark, manufacturer, or otherwise, does not necessarily constitute or imply its endorsement, recommendation, or favoring by the United States Government or any agency thereof or its contractors or subcontractors. The views and opinions of authors expressed herein do not necessarily state or reflect those of the United States Government or any agency thereof. 


\section{Initial Configuration of Acceleration for the IDS-NF Neutrino Factory}

J. Scott Berg

Brookhaven National Laboratory

September 20, 2011

\section{Requirements}

The IDS-NF neutrino factory acceleration system must accelerate the muon beam coming out of cooling to a total energy of $25 \mathrm{GeV}$. The parameters of the beam being accelerated are given in Table 1. While a certain fraction of the beam will be lost early in acceleration, losses of the beam that falls within the acceptance (see Table 1) should be dominated by decay losses (as opposed to dynamic losses or losses on apertures). Decay losses will be kept small, and the cost of the machine will be reduced to the extent possible, consistent with the above requirements. There is a tradeoff between machine cost and the amount of decay losses, and a reasonable compromise will be made between them.

Both muon signs will be accelerated simultaneously.

\section{RF Systems}

The beam will be arriving from the cooling channel in a $201.25 \mathrm{MHz}$ bunch train. Our RF frequency must therefore be an integer multiple of that frequency. Unlike in the bunching, phase rotation, and cooling, we do not require that the RF cavities be in a high magnetic field, so we will choose superconducting RF cavities to reduce the peak RF power that must be delivered to them.
Initially, the large beam aperture and large longitudinal emittance will require the use of the lowest possible frequency (201.25 MHz). While in principle, one could use higher RF frequencies for later stages, we have chosen not to do so because beam loading will begin to impact the beam dynamics, and current designs indicate that we could have problems with physical aperture if $402.5 \mathrm{MHz}$ cavities were used.

The cavity designs will be based on the $\mathrm{Nb}$ on $\mathrm{Cu}$ cavities described in Study II [OPZ+01], whose parameters are given in Table 2. Experiments have achieved a significant fraction of the desired gradient (11 MV/m, compared to a desired gradient of $17 \mathrm{MV} / \mathrm{m}$ ) [GBH+03], and that was only limited by the power limitation of the input coupler. This limitation resulted from a larger than expected $Q$-slope, which we expect to be remedied by better methods for forming and treating the $\mathrm{Nb}$ surface. Fields as high as

Table 1: Parameters of the beam being accelerated. Acceptances are normalized, and are defined in an appendix to this document. The number of particles only counts those within the acceptance.

\begin{tabular}{lr}
\hline \hline Initial total energy & $244 \mathrm{MeV}$ \\
Final total energy & $25 \mathrm{GeV}$ \\
Transverse acceptance & $30 \mathrm{~mm}$ \\
Longitudinal acceptance & $150 \mathrm{~mm}$ \\
Initial particles per bunch train & $3.3 \times 10^{12}$ \\
Bunch trains per pulse & 3 \\
Pulses per second & 50 \\
\hline \hline
\end{tabular}


Table 2: Properties of two types of RF cavities [OPZ+01]. Values are at maximum design gradient. Single cell cavities have energy gain, stored energy, and input power cut in half.

\begin{tabular}{lrr}
\hline \hline RF Frequency (MHz) & 201.25 & 201.25 \\
Cells/cavity & 2 & 2 \\
Aperture diameter (mm) & 300 & 460 \\
Energy gain (MeV) & 25.5 & 22.5 \\
Stored energy (J) & 2008 & 1932 \\
Input power (MW) & 1016 & 980 \\
RF on time (ms) & 3 & 3 \\
Loaded $Q$ & $10^{6}$ & $10^{6}$ \\
\hline \hline
\end{tabular}

$0.1 \mathrm{~T}$ will be permitted at the cavity surface [GBH+03].

\section{Stages: Motivation}

Acceleration will occur in a number of stages. Different types of accelerators will be used at each stage so as to have the lowest cost system for each energy range. Because our accelerator costs are usually dominated by the cost of the RF systems, a more cost-effective system will generally have the beam make more passes through the RF cavities.

\section{FFAG}

The most straightforward way to get a large number of turns in an accelerator is to use a synchrotron. However, since the rate at which magnet fields can change is limited, a synchrotron have an unacceptably large number of decays for the low energies we are dealing with, so is ruled out. Instead, a fixed field alternating gradient accelerator (FFAG) can be used.

The fields in the magnets of an FFAG do not vary during the acceleration cycle, which means that the position of the beam changes as the beam accelerates, as does the time of flight. The position variation leads to large magnet apertures, and magnet costs can start to dominate the machine cost if these are not kept under control. Unlike the other acceleration stages, the FFAG magnet and RF costs will be similar due to this large aperture and the ability of the machine to have a large number of turns. The time of flight variation causes the beam to lose synchronization with the RF, since the short acceleration time combined with the use of high$Q$ RF prevents the RF frequency from being varied during the acceleration cycle. The phase slip due to the time of flight variation ultimately limits the number of turns in such an accelerator.

To minimize the required aperture in the magnets and the variation in the time of flight, a particular type of FFAG known as a linear nonscaling FFAG is used. It incorporates only linear magnets to give a sufficiently large transverse dynamic aperture [MJ97,JWG99]. In comparison to a more traditional (scaling) FFAG design $[\mathrm{SKJ}+56]$, it has a reduced transverse aperture and time of flight variation.

As the energy is reduced, the FFAG becomes more expensive. As the energy is reduced, the geometric emittance increases. This prevents a reduction in cell lengths, and thus as the dispersion increases as we try to reduce the circumference as the energy is reduced. Since the circumference cannot be reduced linearly with momentum and the time of flight variation increases due to the increased dispersion, the amount of RF cannot be reduced linearly with the momentum while keeping a high average gradient. The result is higher magnet costs (due to the larger apertures) and higher RF costs per amount of acceleration. Additionally, there is a nonlinear coupling of the transverse amplitude to the time of flight, leading to an effective longitudinal emittance growth [Mac06,Ber07]. These effects lead one to choose a different type of accelerator for lower energy stages.

\section{$\boldsymbol{R L A}$}

A recirculating linear accelerator (RLA) consists of one or more linacs connected by a number of 
Table 3: Stages for the initial acceleration configuration, specifying the design total energy for the entrance and exit of each stage.

\begin{tabular}{lr}
\hline \hline Stage & Total Energy $(\mathbf{M e V})$ \\
\hline Linac & 244 \\
\hline RLA I & 900 \\
\hline RLA II & 3200 \\
\hline FFAG & 12600 \\
\hline \hline
\end{tabular}

arcs. The beam makes multiple passes through the linac, entering and exiting the linac via a different arc at each pass. This has the advantage that the beam can be kept synchronized to the RF on each pass simply by tuning the length of each arc. The arcs are small compared to those in the FFAG because they only have to transmit a single energy.

The challenge of an RLA is that is must direct the beam into a different arc on each pass. Since the beam has a large energy spread and transverse emittance, there must be significant energy separation between the passes. Additional space for hardware such as magnet coils and iron must be allowed between the beams of different energies. This limits the number of turns one can achieve in an RLA to approximately 4, which is the motivation for using an FFAG at higher energies where they can achieve more turns.

At lower energies, two problems appear. First, the large relative energy spread and geometric emittance make separation and bending of the beam difficult. Second, the velocity variation of the beam with energy means that the phases of the linac cavities cannot be synchronized for both the first pass and subsequent passes. This requires us to use a single pass linac to accelerate from the lowest energies.

\section{Initial Configuration}

The aforementioned considerations have led to four stages of acceleration, whose energy ranges are given in Table 3. Transfer lines will be designed to match transversely (and longitudinally if necessary) between the stages.

\section{Linac}

The linac will be solenoid focused. It will have two types of cells. The linac will start with $3 \mathrm{~m}$ cells containing a single cell cavity. Once the beam size has been reduced sufficiently, the linac will use $5 \mathrm{~m}$ cells containing a double cell cavity to achieve better average RF gradient. The cavities are the large aperture cavities of Table 2.

The RF phase will start far off crest to provide longitudinal focusing for large longitudinal emittance. The phase will steadily move closer to crest as the energy of the beam increases.

\section{RLAs}

The RLAs will have a dogbone shape [BJS01]. For a given amount of RF the dogbone configuration gives a better separation than a racetrack configuration [Pal04].

The beam will be injected into the center of the linac so that the first pass will have less RF phase slippage than it would if it traversed the entire linac [Pal04]. The beam will be injected using a pair of chicanes (one for each sign) such that for higher energy passes in the linac, the orbits close [Pal04]. There will not be any quadrupoles between the dipoles in the linac that separate and recombine the beam at injection.

Each RLA will have 4.5 passes (the half pass being from injection to encountering the first arc). The beam will be taken from the end of the linac on the final pass.

The transverse lattice will be FODO throughout the RLAs. 
Each linac drift will contain a single double cell large aperture cavity for the first RLA, and two double cell small aperture cavities for the second RLA (see Table 2 for parameters).

The arcs will be designed to give zero dispersion in the linac.

Every arc in the RLA will have the same number of cells. This results in a beta function that rises with energy, matching the beta function rise with energy in the linac. This should simplify matching to the linac, and possibly eliminate the need to for sextupoles to correct chromatic aberrations in the matching. The quadrupole strengths in the linac will be used to match the beam to the arcs at each energy. This allows all arcs to use a consistent design for dispersion suppression.

At each end of the linac, there will be a focusing quadrupole (at the linac side), followed by a horizontal separation dipole, followed by a defocusing quadrupole (at the arc side). This configuration amplifies the separation at the switchyard if that defocusing quadrupole encompasses multiple beam signs and/or passes.

Since the dogbone arcs cross each other, the beams will be separated vertically. They will be separated and recombined at the switchyard side of the crossing, and left separated between the two crossings within the arc (vertical dispersion will be zero in that region).

Arc lengths and RF phase will be adjusted to give some level of synchrotron oscillation. They will also be adjusted to accomplish longitudinal matching between stages.

\section{FFAG}

The FFAG ring will consist of nearly identical triplet cells in an FDF configuration. The triplet configuration allows sign symmetric injection and extraction. A FODO cell would also allow this but is more costly [BM10]. Magnets in the triplet will be combined-function superconducting magnets. The drift between magnets in the triplet will be $75 \mathrm{~cm}$, and the long drift will be $5 \mathrm{~m}$. The latter is needed to permit the septum to be sufficiently long to keep the stray fields reasonable.

Injection and extraction will occur at opposite ends of the ring. Kickers will be shared for both signs. No RF cavities will be in the injection or extraction regions. Injection will be from the inside, extraction to the outside. Magnets in the injection and extraction regions may have somewhat larger aperture than those in the main part of the ring, but will have their fields so that, to the extent possible, they have an identical effect on the beam identical to that of the standard magnets. The septa will be superconducting. Kicker fields will be limited to $0.1 \mathrm{~T}$.

Each cell will contain a single double-cell small aperture cavity (see Table 2). Sufficient power will be supplied to the cavities to restore the voltage to the same value for each bunch train.

\section{Acknowledgements}

The starting point for the initial configuration is the design specified in the Interim Design Report (IDR) [IDS11] of the International Design Study for the Neutrino Factory (IDS$\mathrm{NF}$ ). Issues identified in the linacs and RLAs when writing the IDR led to modifications of the configuration which were decided upon at the MAP Acceleration Workshop in April 2011 $[\mathrm{BBB}+11]$.

This manuscript has been authored by employees of Brookhaven Science Associates, LLC under Contract No. DE-AC02-98CH10886 with the U.S. Department of Energy. The United States Government retains a non-exclusive, paid-up, irrevocable, world-wide license to publish or reproduce the published form of this 
manuscript, or allow others to do so, for United States Government purposes.

\section{Appendix: Acceptance Definition}

The goal of this acceptance definition is to define an emittance-like quantity which expresses the maximum particle amplitude that the beamline is required to transmit. We wish the shape of the transmitted phase space to be ellipsoidal (as opposed to a tensor product of ellipses, or a tensor product of intervals).

First assume that at every point along the beamline, there is a closed orbit $\boldsymbol{z}_{0}(s)$ (a 6 dimensional phase space vector) and a symplectic matrix $A(s)($ a $6 \times 6$ matrix $)$ which I will call the normalizing matrix. For some cases (such as the FFAG), $s$ may depend on the turn number. The phase space vectors should contain position and canonical momentum (not momentum scaled by a reference momentum) in the transverse plane, and canonical energy (or energy deviation, but not relative energy deviation) and time in the longitudinal plane.

In a ring with no average acceleration, the closed orbit would be the phase space point that comes back to itself. As for the normalizing matrix, say that $A(s)$ transforms 6 dimensional vector $\left(q_{1}, p_{1}, q_{2}, p_{2}, q_{3}, p_{3}\right)$ to $\boldsymbol{z}-\boldsymbol{z}_{0}(s)$. Then in a ring, to linear order, if $\boldsymbol{z}$ is the phase space vector of a particle, the corresponding quantities $q_{k}^{2}+p_{k}^{2}$ will each remain constant as $\boldsymbol{z}$ is transported from one turn to the next. These quantities are the action variables (again, to linear order). For a general section of beamline where the transfer matrix between $s_{0}$ and $s_{1}$ is $M$, then $A\left(s_{1}\right)=M A\left(s_{0}\right)$. Note that $A$ can be multiplied on the right hand side by a matrix consisting of three rotation blocks on the diagonal, and will still have the necessary properties.
To give a concrete example for clarification, for decoupled transverse motion, the top $2 \times 2$ block of $A$ would be

$$
\left[\begin{array}{cc}
\sqrt{\beta / p_{0}} & 0 \\
-\alpha \sqrt{p_{0} / \beta} & \sqrt{p_{0} / \beta}
\end{array}\right]
$$

where $p_{0}$ is a reference momentum and $\alpha$ and $\beta$ are the Courant-Snyder lattice functions. The addition of $p_{0}$ from the usual definition results from our use of momentum without scaling by a reference momentum, and will generate normalized acceptances.

Say that the normalized acceptances in the three planes are $a_{k}$ (in $\mathrm{m}$ ). Then we say that a particle is within the acceptance if

$$
\sum_{k=1}^{3} \frac{q_{k}^{2}+p_{k}^{2}}{a_{k}}<m c
$$

where $m$ is the muon mass and $c$ is the speed of light, after applying the matrix $A^{-1}(s)$ to its phase space coordinates subtracted from the closed orbit. Coupling is generally sufficiently weak that it is straightforward to determine which planes are transverse (assigned index 1 and 2 , and for our case have the same value for $a_{k}$ of $30 \mathrm{~mm}$ ) and which are longitudinal.

Particles that are within the acceptance will be transmitted through the entire acceleration chain, in the sense that losses of these particles will be significantly less than losses occurring due to decays. We make no assertion about particles that are not within the acceptance.

\section{Appendix: Initial Designs}

I present designs for two of the subsystems described above, namely the linac and the FFAG. These designs are not part of the initial configuration, and are only presented here to give a reference to the existing designs. No assertion is made that they satisfy all of the 
Table 4: Lengths $(\mathrm{cm})$ of lattice elements in the cells of the initial linac design.

\begin{tabular}{lrr}
\hline \hline & Short & Long \\
\hline Drift & 20.5174 & 46.0348 \\
Solenoid & 100.0000 & 100.0000 \\
Drift & 50.0000 & 100.0000 \\
Cavity & 74.4826 & 148.9652 \\
Drift & 30.0000 & 80.0000 \\
\hline & 300.0000 & 500.0000 \\
\hline \hline
\end{tabular}

Table 5: Solenoid fields and cavity phases for the short cells of the initial linac design, in sequence. The crest has a phase of zero, and a positive phase is longitudinally focusing. Precision is for reproducibility in simulations, and does not reflect required field tolerances.

\begin{tabular}{lr}
\hline \hline Field (T) & Phase (rad) \\
\hline-1.05992 & 1.2800 \\
+1.07579 & 1.2570 \\
-1.09286 & 1.2340 \\
+1.11112 & 1.2100 \\
-1.13062 & 1.1870 \\
+1.15129 & 1.1640 \\
-1.17311 & 1.1400 \\
+1.19613 & 1.1170 \\
-1.22027 & 1.0930 \\
+1.24558 & 1.0700 \\
-1.27199 & 1.0470 \\
+1.29948 & 1.0230 \\
-1.32809 & 0.9997 \\
+1.35709 & 0.9764 \\
-1.38781 & 0.9530 \\
+1.41956 & 0.9298 \\
-1.45232 & 0.9065 \\
+1.48607 & 0.8843 \\
-1.52078 & 0.8603 \\
+1.55644 & 0.8373 \\
-1.59302 & 0.8145 \\
+1.63050 & 0.7917 \\
-1.66885 & 0.7690 \\
+1.34261 & 0.7465 \\
\hline \hline
\end{tabular}

criteria for the initial configuration, though only minor modifications should be required for the designs to do so.
Table 6: Solenoid fields and cavity phases for the long cells of the initial linac design, in sequence.

\begin{tabular}{lr}
\hline \hline Field (T) & Phase (rad) \\
\hline-1.46627 & 0.71480 \\
+1.43422 & 0.66780 \\
-1.50114 & 0.64130 \\
+1.56987 & 0.60520 \\
-1.64032 & 0.56960 \\
+1.71237 & 0.53430 \\
-1.78591 & 0.50000 \\
+1.86084 & 0.46600 \\
-1.93704 & 0.43260 \\
+2.01442 & 0.39980 \\
-2.09286 & 0.36760 \\
+2.17229 & 0.33610 \\
-2.25258 & 0.30530 \\
+2.33365 & 0.27510 \\
-2.41542 & 0.24570 \\
+2.49779 & 0.21700 \\
-2.58068 & 0.18900 \\
+2.66402 & 0.16170 \\
-2.74773 & 0.13520 \\
+2.83175 & 0.10950 \\
-2.91601 & 0.08453 \\
+3.00044 & 0.06035 \\
-3.08501 & 0.03694 \\
+3.16965 & 0.01431 \\
-3.25432 & 0.00500 \\
+3.33898 & 0.00000 \\
\hline \hline
\end{tabular}

This design is from a lattice file received from Alex Bogacz on September 14, 2011. It is essentially the design given in [KBA+11], which supersedes that of [IDS11]. There are two types of cells with the same basic structure, given in Table 4.

The design as given accelerates to a total energy of $913.485 \mathrm{MeV}$. It assumes the large aperture Study II cavities. The long cells have a two cell cavity; the short cells have a one cell cavity. Cavity phases for each cell are given in Tables 5 and 6. Calculations include corrections for transit time factor and include transverse focusing from RF.

\section{Linac}


Table 7: Parameters for the magnets in the initial FFAG design. The angle and shift are defined such that on entering the magnet, the coordinate system rotates by half the angle then shifts by the shift value, and on exiting the magnet the coordinate system shifts by the negative of the shift value then rotates by half the angle again. The angle and the shift would be positive to give the usual sagitta correction for a dipole magnet with a positive bend angle. Precision is for reproducibility in simulations, and does not reflect required field tolerances. Aperture radius and pole tip fields are derived from the lattice definition, required acceptances, and multiplying the aperture required for the beam by 1.3 .

\begin{tabular}{lrr}
\hline \hline & D & F \\
\hline Length (m) & 1.994466 & 0.965155 \\
Angle (mrad) & +147.626 & -26.924 \\
Shift (mm) & +39.012 & +14.371 \\
Field (T) & +4.43410 & -1.43705 \\
Gradient (T/m) & -14.0598 & +18.8800 \\
Aperture radius (mm) & 130 & 160 \\
Pole tip field (T) & 6.3 & 4.5 \\
\hline \hline
\end{tabular}

The solenoids have a $23 \mathrm{~cm}$ radius aperture. Their strengths are given in Tables 5 and 6 . Calculations include a focusing correction at the magnet ends.

\section{FFAG}

This design is a slight update of that given in [BAW+11], and supersedes the design in [IDS11].

The FFAG lattice consists entirely of FDF triplet cells. The lattice has 67 cells, with a $5 \mathrm{~m}$ long drift and a $75 \mathrm{~cm}$ drift between the triplet magnets. Each magnet is a combined function magnet. The magnet properties are given in Table 7.

We will use double cell small aperture cavities described in Table 2: Properties of two types of RF cavities [OPZ+01]. Values are at maximum design gradient. Single cell cavities have energy gain, stored energy, and input power cut in half.Table 2, with a single cavity in most long drifts. There will be 50 cavities in the ring, with
Table 8: Magnet parameters for the injection/extraction system. The pattern is the arrangement and polarity of kickers in sequential cells: - or + for inward or outward kicks, respectively, and $\bigcirc$ for an empty cell. Parameters are taken from [BAW+11], and were chosen to work with the design in [IDS11].

\begin{tabular}{lrr}
\hline \hline & Injection & Extraction \\
\hline Kickers & 2 & 4 \\
Pattern & $-\mathrm{O}$ & $++\mathrm{O}++$ \\
Kicker field (T) & 0.089 & 0.067 \\
Septum field (T) & 0.92 & 1.76 \\
Magnet length (m) & 4.4 & 4.4 \\
\hline \hline
\end{tabular}

a total RF voltage of $1212.571 \mathrm{MV}$. This will allow acceleration in around 11.6 turns.

Injection and extraction systems will be at opposite ends of the ring. The same kickers will be used to inject both signs, similarly for extraction. Septa (one for each sign) will be in the cells just outside the sequence of cells containing kickers. Table 8 gives the parameters for the injection system. Magnets in the injection and extraction regions will need to have slightly increased apertures to accommodate the injection and extraction oscillations. The design must try to minimize the difference between these magnets and the magnets in the other regions.

\section{References}

[BAW+11] J. Scott Berg, Morteza Aslaninejad, Jaroslaw Pasternak, Holger Witte, Neil Bliss, Michael Cordwell, Thomas Jones, Alan Muir, David Kelliher, and Shinji Machida, "A Non-Scaling Fixed Field Alternating Gradient Accelerator for the Final Acceleration Stage of the International Design Study of the Neutrino Factory," to appear in the proceedings of the 2nd International Particle Accelerator Conference (IPAC 2011), San Sebastián, Spain (2011). 
$[\mathrm{BBB}+11]$ J. Scott Berg, Kevin Beard, Alex Bogacz, and Ajit Kurup, "MAP Acceleration Meeting, April 2011: IDS-NF Linac and RLA Design" (2011). http://www.cap.bnl.gov/mumu/conf/ accel-110413/

[Ber07] J. Scott Berg, "Amplitude dependence of time of flight and its connection to chromaticity," Nucl. Instrum. Methods A 570 (2007) 15.

[BJS01] J. Scott Berg, Carol Johnstone, and Don Summers, "Dogbone Geometry for Recirculating Accelerators," in Proceedings of the 2001 Particle Accelerator Conference, edited by P. Lucas and S. Webber (IEEE, Piscataway, NJ, 2001) 3323.

[BM10] J. Scott Berg and Shinji Machida, "FFAG Designs for the International Design Study for the Neutrino Factory," in Proceedings of PAC09, Vancouver, BC, Canada (2010) 657.

[GBH+03] R. L. Geng, P. Barnes, D. Hartill, H. Padamsee, J. Sears, S. Calatroni, E. Chiaveri, R. Losito, and H. Preis, "200 $\mathrm{MHz} \mathrm{Nb}-\mathrm{Cu}$ Cavities for Muon Acceleration," in Proceedings of the $11^{\text {th }}$ Workshop on RF Superconductivity, Lübeck/Travemünder, Germany (2003) 531.

[IDS11] S. Choubey et al. (The IDS-NF Collaboration), "Interim Design Report," IDSNF-020 (2011). https://www.ids-nf.org/

[JWG99] C. Johnstone, W. Wan, and A. Garren, "Fixed Field Circular Accelerator Designs," in Proceedings of the 1999 Particle Accelerator Conference, New York, 1999, edited by A. Luccio and W. MacKay (IEEE, Piscataway, NJ, 1999) 3068.

[KBA+11] A. Kurup, C. Bontoiu, M. Aslaninejad, J. Pozimski, A. Bogacz, V. S. Morozov, Y. R. Roblin, and K. B. Beard,
"The Muon Linac for the International Design Study for the Neutrino Factory," to appear in the proceedings of the 2nd International Particle Accelerator Conference (IPAC 2011), San Sebastián, Spain (2011).

[Mac06] Shinji Machida, "Longitudinal emittance blowup in fixed field alternating gradient muon accelerators," Phys. Rev. ST Accel. Beams 9 (2006) 104002.

[MJ97] F. E. Mills and C. Johnstone, "Linear Orbit Recirculators," in 4th International Conference on Physics Potential \& Development of $\mu+\mu$-colliders, December 10 12, 1997, San Francisco, California: Transparency Book, 693-698.

[OPZ+01] S. Ozaki, R. B. Palmer, M. S. Zisman, and J. C. Gallardo, "Feasibility Study-II of a Muon-Based Neutrino Source," BNL-52623 (2001).

[Pal04] R. B. Palmer, "Accelerator from Cooling to FFAGs," talk at the Neutrino Factory and Muon Collider Workshop on Ring Coolers and Emittance Exchange (2004). http://www.cap.bnl.gov/mumu/conf/ E-EX-040121/.

[SKJ+56] K. R. Symon, D. W. Kerst, L. W. Jones, L. J. Laslett, and K. M. Terwilliger, "Fixed-Field Alternating-Gradient Particle Accelerators," Phys. Rev. 103 (1956) 1837. 